\title{
Corrigendum: Inflammatory Cytokine Expressions of the Subacromial Bursitis and Glenohumeral Joint Synovitis in the Patients with Full Thickness Rotator Cuff Tear
}

Sung-Kyu Kim, Hyung-Nam Kim, Eun-Sun Moon, Keun-Young Lim, Nam-Young Cho, Myung-Sun Kim

Department of Orthopaedic Surgery, Chonnam National University College of Medicine, Gwangju, Korea

https://doi.org/10.5397/CiSE.2011.14.2.172

Clin Shoulder Elbow 2011;14(2):172-178

This correction is being published to correct the order of the first and second authors' names in the above article.

Before correction: Sung-Kyu Kim, Hyung-Nam Kim, Eun-Sun Moon, Keun-Young Lim, Nam-Young Cho, Myung-Sun Kim

After correction: Hyung-Nam Kim, Sung-Kyu Kim, Eun-Sun Moon, Keun-Young Lim, Nam-Young Cho, Myung-Sun Kim

This article was corrected online. The authors apologize for this error and any inconvenience that it may have caused. 